\title{
Performance Analysis of Hybrid ALOHA/CDMA RFID Systems with Quasi-decorrelating Detector in Noisy Channels
}

\author{
Hoang Trung Tuyen ${ }^{1,2}$, Dao Van Hieu ${ }^{1}$, Phan Xuan $\mathbf{V u}^{1}$, Nguyen Thanh Chuyen ${ }^{1}$ \\ ${ }^{1}$ School of Electronics and Telecommunications, Hanoi University of Science and Technology, Hanoi, \\ Vietnam \\ 2 Military Science Academy, Hanoi, Vietnam
}

Correspondence: Nguyen Thanh Chuyen, chuyen.nguyenthanh@hust.edu.vn

Communication: received 6 August 2018, revised 14 December 2018, accepted 4 March 2019

Online publication: 30 June 2019, Digital Object Identifier: 10.21553/rev-jec.212

The associate editor coordinating the review of this article and recommending it for publication was Dr. Diep Nguyen.

\begin{abstract}
In this paper we investigate the performance of a hybrid Aloha/CDMA radio frequency identification (RFID) system with quasi-decorrelating detector (QDD). Motivated by the fact that the QDD outperforms the conventional decorrelating detector (DD) in noisy network scenarios, we study and propose using QDD as one of the most promising candidates for the structure of RFID readers. Performance analysis in terms of bit error rate and the RFID system efficiency is considered considering CDMA code collision and detection error. Computer simulations are also performed, and the obtained results of QDD-based structure are compared with those of DD-based one to confirm the correctness of the design suggestion in different practical applications of tag identification and missing-tag detection.
\end{abstract}

Keywords- Dense RFID, identification, de-correlating detector, quasi-decorrelating detector, code collision, detection errors, system efficiency.

\section{INTRODUCTION}

Radio Frequency IDentification (RFID) is well known as a wireless technology that automatically and efficiently identifies the objects. For years, RFID has been widely implemented in many practical applications for years such as security check, object tracking, supply chain management, medical management, and environment monitoring [1-5]. The technology also plays a key role in the access layer of the Internet of Things (IoT) paradigm thanks to its advantages of low cost, simplicity, and no-line-of-sight radio transmission [6, 7]. A typical RFID system consists of a reader and a number of tags each of which is represented by a unique 96-bit (or 128-bit) identity (ID) [8]. The reader tries to detect and monitor all the tags' ID quickly and reliably $[3,5]$.

Although RFID technology has provided numerous benefits for automatically identifying objects in many practical applications, it still has problems associated with tag collision and missing-tag detection. Tag collision happens when multiple tags transmit their signal simultaneously $[4,9,10]$. In this case, the signals collide at the reader and the tags would not be successfully identified. Besides, missing-tag detection is to find out whether any tag is missing, which plays an important role in industrial applications. In oder to monitor a number of tags, the reader is able to know the presence and absence of tags. This helps to detect the missing tag event and reduces the economic loss caused by shoplifting, employee theft, and vendor fraud [11, 12].

In order to cope with the tag collision and missingtag event detection, many communications/access pro- tocols have been designed. They are based on different multiple access techniques, which schedule and control the order of each tag's transmission. The techniques could be, for examples, Frequency Division Multiple Access (FDMA), Space Division Multiple Access (SDMA), Time Division Multiple Access (TDMA), and Code Division Multiple Access (CDMA) [13, 14]. More specifically, FDMA-based protocols use different frequency bands for different tags' responses, while SDMA-based ones adopt multiple-antenna techniques or power control algorithms to mitigate the collision. They, thus, might have a higher performance, yet are extremely complex and lead to the higher cost as well [1517]. In CDMA-based ones, each tag is assigned with a pseudo-code so that multiple tags could be successfully identified at the same time without collision with a suitable design of multi-user detector at the reader. CDMA technique can be even implemented in a passive tag whose structure is very simple without internal power [14], which will be very promising in the context of the IoT where the number of devices is very large. In TDMA-based protocols, tags transmit signal in time slots controlled by the reader. Aloha-based protocol [18] is adopted as the Medium Access Control (MAC) layer standard in the context of RFID [19]. Thank to the simplicity and efficiency, the hybrid Aloha/CDMA-based RFID i.e., CDMA tags randomly respond in time slots, has attracted many research topics in dense network scenarios recently, and is also our focus in this work. In this hybrid scheme, decorrelating detector (DD) has been first supposedly implemented in the reader structure [20] thanks to its advantages in mitigating 
Multi-Access Interferences (MAI) caused by the imperfectness of the pseudo-codes. The detector has been also studied in designing missing-tag event detection protocols [21]. Nevertheless, the DD might also enhance the background noise according to [22], and thus, might degrade the performance of RFID systems.

In this paper, we therefore re-study the performance of hybrid ALOHA/CDMA-based RFID systems with another multi-user detector, namely Quasidecorrelating detector (QDD) [23]. QDD has been considered as a truncated multi-stage version of DD, which can overcome the disadvantage of the noise enhancement in DD. Then, by assuming the suggested design of QDD installed into the reader structure, we theoretically analyze the tag identification performance of the hybrid systems under both code collision [24] (when transmitted tags share the same code) and detection errors [8] (due to wireless channel impairments). We also study the impact of QDD on the performance of missing-tag event detection, which is one of the most important issues in the context of RFID industry. The main contributions of our work are as follows:

- We first describe the performance of the QDD in the context of RFID where each tag is assigned with a Gold code sequence. By adopting the standard Frame Slotted Aloha (FSA) at MAC layer, we theoretically analyze the performance of RFID tag identification in terms of system efficiency. The performance is studied not only in a perfect channel model but also an imperfect one where both the code collision and the detection error are taken into account.

- We consider the impact of QDD on the performance missing-tag event detection in terms of false alarm and false detection. Two performance metrics i.e., false alarm and false detection rates are derived and evaluated with different system parameters settings to describe the reliability of the detection protocols.

- Computer simulations are performed using MonteCarlo method to prove the correctness of our analysis. The obtained results of both identification and missing-tag detection protocols with QDD structure are compared with conventional ones using DD to confirm the efficiency of the design suggestion. The comparison is believed to be useful for system designers in terms of protocol selection and network structures in the context of RFIDbased IoT.

This paper is organized as follows: In Section 2, the considered RFID system, transmission channel model, and the conventional DD are described. Section 3 focuses on the QDD and the performance analysis of RFID efficiency. Section 4 presents the numerical results and discussion. Finally, conclusions are drawn in Section 5.

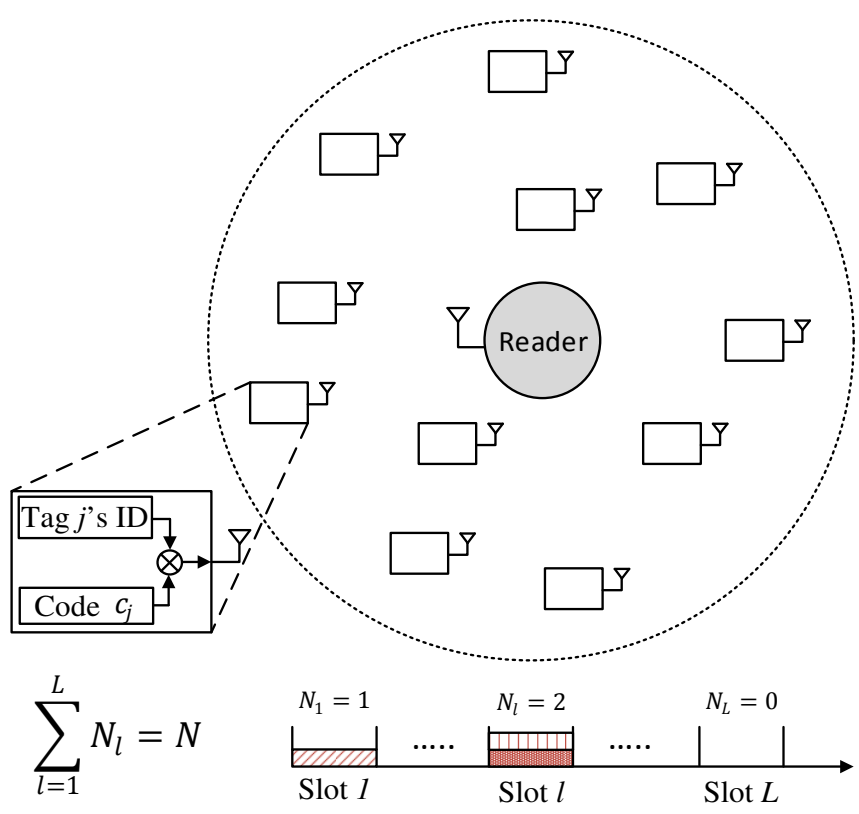

Figure 1: CDMA-based RFID system with FSA protocol.

\section{System Description and Conventional APPROACH}

\subsection{System Model}

Our considered RFID system consists of a reader and $N$ CDMA tags as shown in Figure 1. Each tag has a unique 96-bit ID, and is randomly assigned with one of the $K=2^{m}+1, m$ is the length of the register, $(K<N)$ Gold codes of the code set $G_{K}$ [14]. Here, Gold codes are considered in our model for simplicity, while other types of code can be implemented in the same way. Let $c_{j}$ denote by the code of the $j$-th tag and different tags might have the same code. The information of a tag is sent to the reader after spreading by the corresponding code.

Frame Slotted ALOHA (FSA) is used in our system as a standard MAC protocol for tag identification. In particular, the reader first broadcasts a message consisting of a frame size $L$ and a random seed $R$ in each reading round. Then, tags randomly respond to the reader their IDs among $L$ slots thank to a hash function of $h(L, R, \mathrm{ID})$ [25]. During each time slot tags might be collided and thus, not detected if they use the same code, which we call code collision. This process of broadcasting the frame of time slots and collecting tags' ID is repeated until all the tags are successfully identified. Here, it is noted that tags do not respond to the reader after being detected. Moreover, if we denote by $N_{l}$ the number of tags in the $l$-th slot $(l=1, \ldots, L)$, we have

$$
\sum_{l=1}^{L} N_{l}=N
$$

\subsection{Transmission Channel Model}

Since we focus on the impact of the background noise on the signal detection at the reader, the transmission 


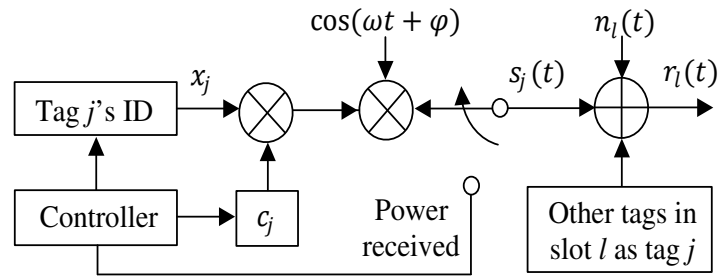

Figure 2: Transmission channel model.

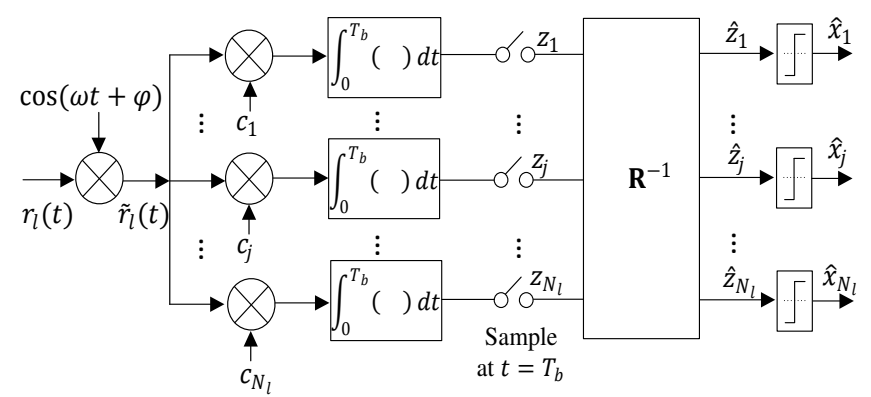

Figure 3: Reader structure with decorrelating detector.

channel model is assumed to be Additive White Gaussian Noise (AWGN) for a simple analysis, as described in Figure 2. Nevertheless, we also investigate the system performance evaluation in a more practical Rayleigh fading one. In particular, the received signal during the $l$-th slot is written as

$$
\begin{aligned}
r_{l}(t) & =\sum_{j \in\left\{N_{l}\right\}} s_{j}(t)+n_{l}(t) \\
& =\sqrt{2 P} \sum_{j \in\left\{N_{l}\right\}} x_{j} c_{j} \cos (\omega t+\varphi)+n_{l}(t),
\end{aligned}
$$

where $r_{l}(t)$ is the received signal. $n_{l}(t)$ is White Gaussian noise with zero-mean and $\sigma^{2}$-variance. $\left\{N_{l}\right\}$ is an index set of $N_{l}$ tags involving in the $l$-th slot. The transmitted signal from the $j$-th tag denoted by $s_{j}(t)$ can be expressed as

$$
s_{j}(t)=\sqrt{2 P} x_{j} c_{j} \cos (\omega t+\varphi),
$$

where $P$ is the transmit power, and is assumed to be the same for every tag. $x_{j} \in\{-1,+1\}$ is the information bit of the $j$-th tag. Code $c_{j}$ is written as

$$
c_{j}=\sum_{a=1}^{L_{c}} c_{a}^{(j)} p\left(t-l T_{c}\right)
$$

where $T_{c}$ is a chip duration, and each chip $c_{a}^{(j)}$ of the code takes either value of +1 or $-1 . p(t)$ is a rectangular pulse i.e.,

$$
p(t)= \begin{cases}1 & \text { if } 0 \leq t \leq T_{\mathcal{c}} \\ 0 & \text { otherwise }\end{cases}
$$

\subsection{Conventional Decorrelating Detector}

In order to detect signal transmission from many tags, multi-user detectors are implemented in the reader. According to [14, 20, 21, 24], DD-based protocol is one of the most efficient solutions for RFID, both in theoretical and experimental aspects, thanks to advan- tages of MAI elimination and simple structure. The DD was first introduced by Lupas and Verdú [26]. Reader structure with DD is presented in Figure 3. The received signal during the $l$-th slot, after removing the carrier, is fed into a set of filters that match with corresponding Gold codes in the slot. Then, output signal of the filters, after sampling, goes to a matrix denoted by $\mathbf{R}^{-1}$ for the MAI elimination. For more specific illustration, the filters' output signal can be expressed in a matrix form as follows

$$
\mathbf{z}=\mathbf{R} \mathbf{x}+\mathbf{n},
$$

where $\mathbf{R}$ is the correlation matrix generated by the corresponding codes. $\mathbf{x}$ is the vector of transmitted information bits, and $\mathbf{n}$ is a vector of White Gaussian noise. The signal vector after MAI elimination denoted by $\hat{\mathbf{z}}$ is then presented as

$$
\hat{\mathbf{z}}=\mathbf{R}^{-1} \mathbf{z}=\mathbf{x}+\mathbf{R}^{-1} \mathbf{n},
$$

where $\mathbf{R}^{-1}=\operatorname{inv}(\mathbf{R})$, is the inversion matrix of $\mathbf{R}$. The estimate of $x$, i.e., $\hat{\mathbf{x}}$ is found as

$$
\hat{\mathbf{x}}=\operatorname{sgn}(\hat{\mathbf{z}})=\operatorname{sgn}\left(\mathbf{x}+\mathbf{R}^{-1} \mathbf{n}\right) .
$$

Here, there are two components at the output of the detector, which are signal information $\mathbf{x}$ and background noise with zero mean and variance equal to the $j j$-th component of the covariance matrix $\sigma^{2} \mathbf{R}^{-1}$.

The bit error probability of the $j$-th tag denoted by $P_{e}^{\mathrm{DD}}(j)$ can be determined by [23]

$$
P_{e}^{\mathrm{DD}}(j)=Q\left(\frac{\sqrt{\mathrm{SNR}_{j}}}{\sqrt{\left(\mathbf{R}^{-1}\right)_{j j}}}\right),
$$

where $Q(\cdot)$ is monotonically decreasing function defined as $Q(x)=\frac{1}{\sqrt{2 \pi}} \int_{x}^{\infty} e^{-t^{2} / 2} d t$ while $\mathrm{SNR}_{j}=$ $E\left[s_{j}^{2}(t)\right] / \sigma^{2}$ is the signal to noise ratio of the $j$-th tag. $\left(\mathbf{R}^{-1}\right)_{j j}$ is the noise enhancement factor. When all the elements of $\mathbf{R}$ are less or equal to 1 , this leads $\left(\mathbf{R}^{-1}\right)_{i j}>1$ due to non-orthogonal of Gold codes. Thus, the performance of DD can be severely degraded due to the enhancement of the noise power. To overcome this problem associated with the inversion matrix, a new matrix is used in Quasi-decorrelating detector (QDD) instead of $\left(\mathbf{R}^{-1}\right)$. QDD has been studied for years and proved as one of the most efficient solutions to cope with this situation, which motivates us to propose this work.

\section{Performance Analysis}

In this section, we first describe the performance of QDD and then, analyze the efficiency of the hybrid ALOHA/CDMA RFID system with QDD under impacts of noisy channels.

\subsection{Quasi-decorrelating Detector (QDD)}

In this subsection, in order to introduce the QDD, we rewrite the matrix $\mathbf{R}=\mathbf{I}-\mathbf{A}$, where $\mathbf{I}$ is the $N \times N$ 
identity matrix and the multi-stage feed-forward matrix denoted by $\mathbf{A}$, can be written

$$
\mathbf{A}=\left[\begin{array}{cccc}
0 & -R_{12} & \ldots & -R_{1 N} \\
-R_{21} & 0 & \ldots & -R_{2 N} \\
\ldots & \ldots & \ldots & \ldots \\
-R_{N 1} & -R_{N 2} & \ldots & 0
\end{array}\right],
$$

where $R_{m n}, m, n \in\{1,2, \ldots, N\}$ is the cross-correlation coefficient of matrix $\mathbf{R}$ [23]. The inverse matrix $\mathbf{R}^{-1}$, can be represented by

$$
\mathbf{R}^{-1}=(\mathbf{I}-\mathbf{A})^{-1} .
$$

Using the geometric series [27], the matrix $\mathbf{R}^{-1}$ in (11) can be expanded

$$
\mathbf{R}^{-1}=(\mathbf{I}-\mathbf{A})^{-1}=\mathbf{I}+\mathbf{A}+\mathbf{A}^{2}+\ldots+\mathbf{A}^{r}+\ldots
$$

If $r$ becomes infinity, $\left(\mathbf{I}+\mathbf{A}+\mathbf{A}^{2}+\ldots+\mathbf{A}^{r}+\ldots\right)$ approaches $\mathbf{R}^{-1}$. Here, we denote by $\mathbf{M}_{r}$ a truncation of (12), with only the first $r+1$ terms retained, i.e.,

$$
\mathbf{M}_{r}=\mathbf{I}+\mathbf{A}+\mathbf{A}^{2}+\ldots+\mathbf{A}^{r},
$$

is described in Figure 4.

In order to mitigate MAI, QDD uses matrix $\mathbf{M}_{r}$ instead of the matrix $\mathbf{R}^{-1}$. The output signal from the filter illustrated in Figure 4, can be determined by

$$
\hat{\mathbf{z}}_{r}=\mathbf{M}_{r} \mathbf{z} .
$$

The decoded information vector $\hat{\mathbf{x}}_{r}$ is

$$
\hat{\mathbf{x}}_{r}=\operatorname{sgn}\left(\hat{\mathbf{z}}_{r}\right) .
$$

The bit error probability of the $j$-th tag using QDD, which is denoted by $P_{e}^{\mathrm{QDD}}(j)$, can be written as (16) [23] where

$$
\mathbf{M}_{r} \mathbf{R}=\left(\mathbf{I}+\mathbf{A}+\mathbf{A}^{2}+\ldots+\mathbf{A}^{r}\right)(\mathbf{I}-\mathbf{A})=\left(\mathbf{I}-\mathbf{A}^{r+1}\right),
$$

and

$$
\begin{aligned}
\mathbf{M}_{r} \mathbf{R} \mathbf{M}_{r}^{T}= & \mathbf{I}+\mathbf{A}+\mathbf{A}^{2}+\ldots+\mathbf{A}^{r} \\
& -\mathbf{A}^{r+1}-\mathbf{A}^{r+2}-\ldots-\mathbf{A}^{2 r+1} .
\end{aligned}
$$

By substituting (17) and (18) into (16), we have (19) where $S_{j}(\mathrm{QDD})=-\mathbf{A}^{r+1}-\mathbf{A}^{r+2}-\ldots-\mathbf{A}^{2 r+1}$. As $r$ becomes infinity, $P_{e}^{\mathrm{QDD}}(j)$ approaches $P_{e}^{\mathrm{DD}}(j)$. Here, it was analytically shown in [23] that $P_{e}^{\mathrm{QDD}}(j)$ is smaller than $P_{e}^{\mathrm{DD}}(j)$ in $(9)$ in noisy channels if the number of feed-forward stage or $r$ is chosen properly, especially when most the correlation coefficients of $\mathbf{R}$ are negative. On the other hand, it was also numerically pointed out in [23] that the computational complexity of QDD was lower than that of DD. DD has to calculate the inverse of the cross-correlation matrix, while QDD only finds a $r$ truncated version of the matrix as described in (17) and (18). As a result, QDD takes smaller numbers of multiplication and addition operations than DD depending on the selection of $r$. When $r \rightarrow \infty$, the performance of QDD is as same as $D D$, while in this work, $r=3$ is selected for computer performance evaluation. Overall, QDD might be another potential solution for multi-tag detection in hybrid ALOHA/CDMA RFID systems.

\subsection{Performance Analysis of Tag Identification Efficiency}

We now analyze the impacts of both code collision (when tags in a slot have the same code) and detection error (caused by wireless channel impairments) on the system efficiency defined as the average number of successfully detected tags over a slot. In particular, if we denote by $\eta$ the system efficiency, it can be computed by

$$
\eta=\sum_{i=1}^{N} P_{\text {aloha }}(i) \sum_{a=0}^{\min (i, K)} a P_{\mathrm{d}}(a \mid i) P_{\mathrm{s}}(a \mid i),
$$

where $P_{\text {aloha }}(i)$ is a probability that $i$ tags among $N$ tags simultaneously transmit their IDs. It follows binomial distribution, and can be calculated as

$$
P_{\text {aloha }}(i)=\left(\begin{array}{c}
N \\
i
\end{array}\right)\left(\frac{1}{L}\right)^{i}\left(1-\frac{1}{L}\right)^{N-i} .
$$

$\sum_{a=0}^{\min (i, K)} a P_{\mathrm{d}}(a \mid i) P_{\mathrm{s}}(a \mid i)$ represents $a$ among the $i$ tags that can be successfully detected in a slot. Here, $P_{\mathrm{d}}(a \mid i)$ is a probability that $a$ tags are not collided, while $P_{\mathrm{S}}(a \mid i)$ is a probability that they are successfully detected. We first derive $P_{\mathrm{d}}(a \mid i)$ by expressing it under the following form

$$
P_{\mathrm{d}}(a \mid i)=P_{\text {cdma }}(a \mid i, K) P_{\text {cdma }}^{\mathrm{c}}(i-a \mid i, K-a),
$$

where $P_{\text {cdma }}(a \mid i, K)$ is a probability that $a$ tags are assigned with $a$ different codes of the $K$ codes, which can be easily written as

$$
P_{\text {cdma }}(a \mid i, K)=\frac{\left(\begin{array}{c}
i \\
a
\end{array}\right)\left(\begin{array}{c}
K \\
a
\end{array}\right) a !(K-a)^{i-a}}{K^{i}} .
$$

$P_{\mathrm{cdma}}^{\mathrm{c}}(i-a \mid i, K-a)$ is a probability that the remaining $(i-a)$ tags are collided with the $(K-a)$ codes, which is recursively calculated as (24) [24] where $i_{a}=i-a$ and $K_{a}=K-a$.

We now determine $P_{S}(a \mid i)$ in (20) by analyzing the effect of background noise on the tag detection. Let's denote $P_{\mathrm{S}}(j)$ a probability that the $j$-th tag is successfully detected, we have

$$
P_{\mathrm{s}}(j)=\left(1-P_{e}^{\mathrm{QDD}}(j)\right)^{M},
$$

where $P_{e}^{\mathrm{QDD}}$ can be obtained from (19). Here, it is also worthy to mention that all $M$ bits of the tag are required correctly received for a successful detection. As a result, $P_{\mathrm{S}}(a \mid i)$ can be found as follows

$$
P_{s}(a \mid i)=\prod_{j \in\{a\}} P_{\mathrm{S}}(j),
$$

where $a$ tags indexed by $a_{i}$. Finally, the system efficiency $\eta$ can be obtained by simply substituting (22) and (26) into (20).

\section{Performance Evaluation and Discussions}

In this section, the performance of the considered RFID systems with QDD is evaluated under different system parameters. The obtained results are also compared 


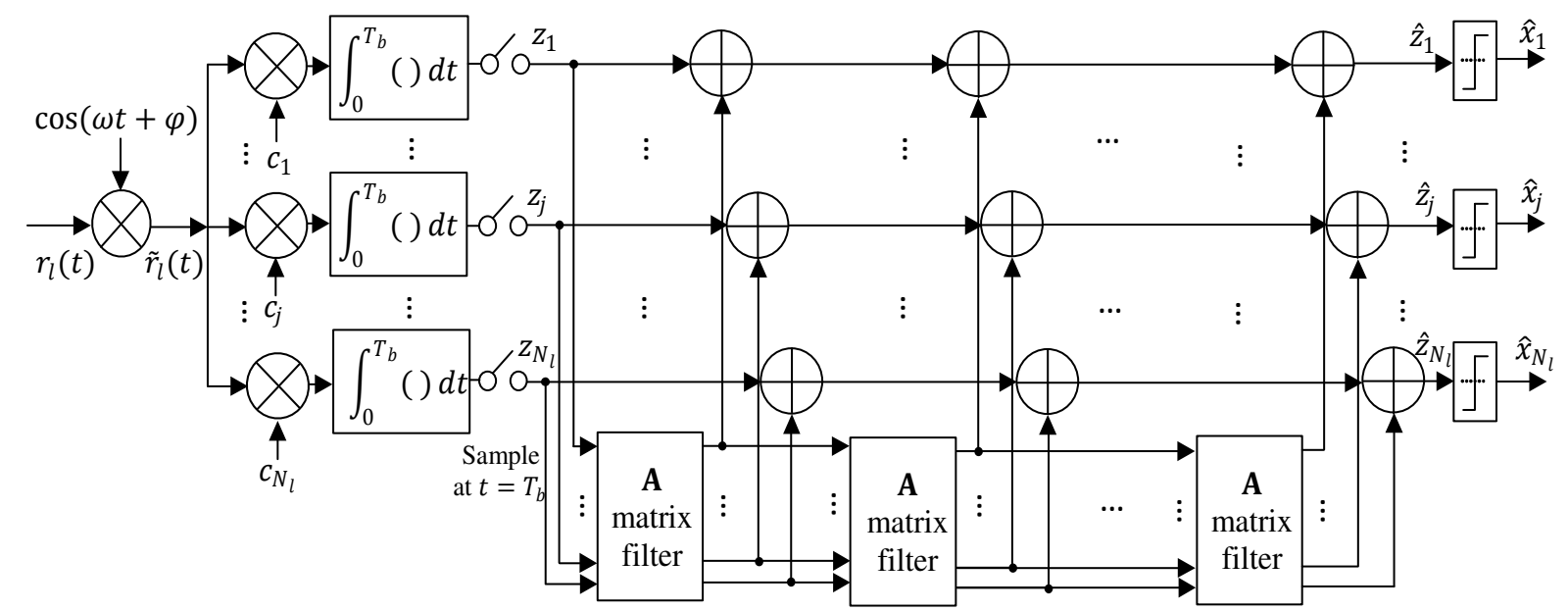

Figure 4: Quasi-decorrelating detector structure.

$$
P_{e}^{\mathrm{QDD}}(j)=\left(\frac{1}{2}\right)^{N-1} \times \sum_{\substack{x \in\{-1,+1\}^{N-1} \\ x_{j}=-1}} Q\left(\frac{\left(\mathbf{M}_{r} \mathbf{R}\right)_{j j} \sqrt{\mathrm{SNR}_{j}}-\sum_{\substack{i=1 \\ i \neq j}}^{N}\left(\mathbf{M}_{r} \mathbf{R}\right)_{j i} x_{i} \sqrt{\mathrm{SNR}_{i}}}{\sqrt{\left(\mathbf{M}_{r} \mathbf{R} \mathbf{M}_{r}^{T}\right)_{j j}}}\right)
$$

$$
P_{e}^{\mathrm{QDD}}(j)=\left(\frac{1}{2}\right)^{N-1} \times \sum_{\substack{x \in\{-1,+1\}^{N-1} \\ x_{j}=-1}} Q\left(\frac{\left[1-\left(\mathbf{A}^{r+1}\right)_{j j}\right] \sqrt{\mathrm{SNR}_{j}}-\sum_{\substack{i=1 \\ i \neq j}}^{N}\left(\mathbf{A}^{r+1}\right)_{j i} x_{i} \sqrt{\mathrm{SNR}_{i}}}{\sqrt{1+(\mathbf{A})_{j j}+\ldots+\left(\mathbf{A}^{r}\right)_{j j}+S_{j}(\mathrm{QDD})}}\right)
$$

$$
P_{\text {cdma }}^{\mathrm{c}}\left(i_{a} \mid i, K_{a}\right)= \begin{cases}1 & \text { if } i_{a}=0 \text { or } K_{a}=0 \\ 1-\left(1-\frac{1}{K_{a}}\right)^{i-1} & \text { if } i_{a}=1 \\ P_{\text {cdma }}^{\mathrm{c}}\left(i_{a}-1 \mid i, K_{a}\right)-\left(1-\frac{1}{K_{a}}\right)^{i-1} P_{\text {cdma }}^{\mathrm{c}}\left(i_{a}-1 \mid i-1, K_{a}-1\right) & \text { if } i_{a} \geqslant 2,\end{cases}
$$

with those of DD-based one to show the effectiveness of the proposed scheme. Monte-Carlo simulations are performed for 1000 iteration runs with Matlab software.

\subsection{System Efficiency}

In Figure 5, we describe the bit-error-rate (BER) performance (both theoretically and simulation) of the QDD and DD with respect to different numbers of tags, for given SNR of $7 \mathrm{~dB}$. Here, it is noted that the SNR can be also set by any other values that can illustrate the effect of noisy channel on the detectors' performance. The Gold code length denoted by $L_{\mathrm{c}}$ is set by 31 chips, while the number of stages in QDD i.e., $r$ is set by 3 . It is observed that the simulation results match with the theoretical ones, validating the analysis. The BER also increases with respect to the increasing of the number tags due to interference. However, the performance of the QDD is better than that of the DD, when the number of tags is large enough $(\geq 10)$. The reason is that the noise has been enhanced in DD under the effect of the code correlation matrix, while in QDD it is mitigated thanks to the stage truncation of the matrix.

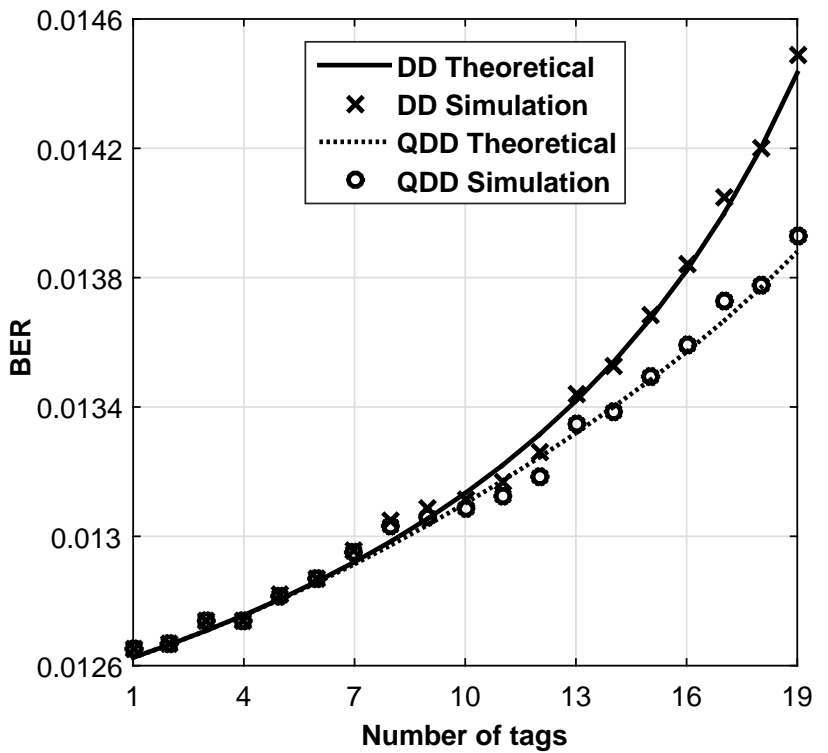

Figure 5: BER performance of QDD and DD detectors with respect to a number of tags, given $L_{\mathrm{C}}=31, \mathrm{SNR}=7$ $\mathrm{dB}, r=3$. 


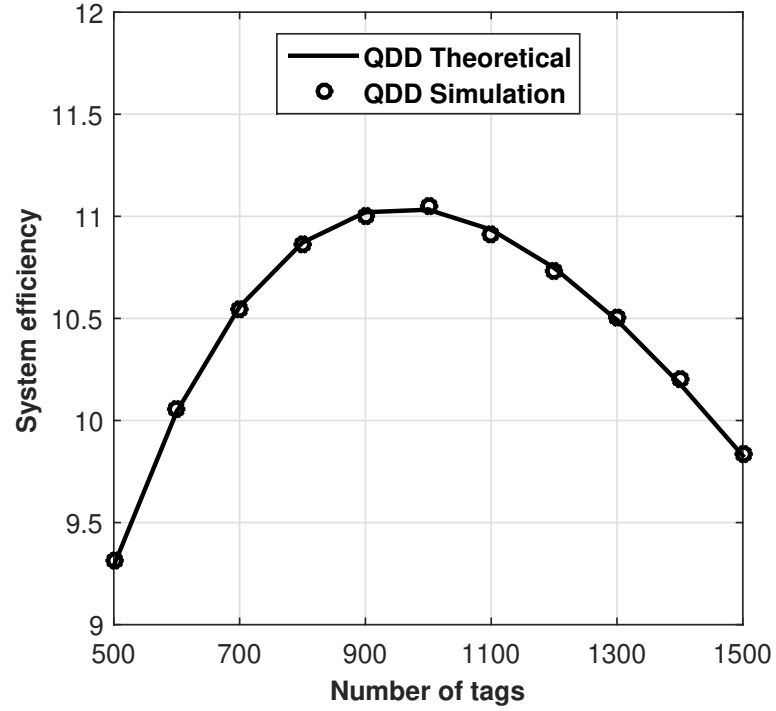

Figure 6: System efficiency with respect to the number of tags, given $L=32, K=30, L_{\mathrm{c}}=30, \mathrm{SNR}=7 \mathrm{~dB}$.

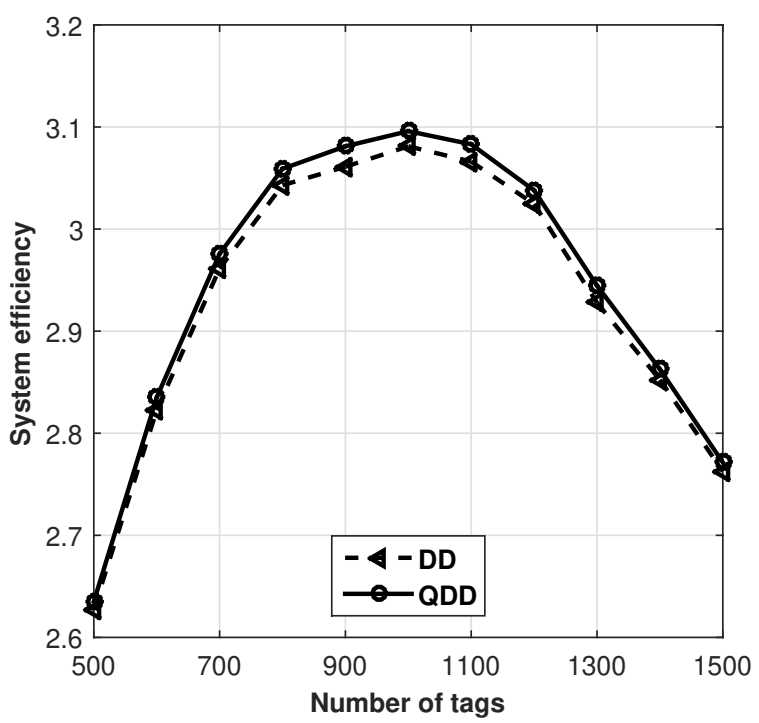

Figure 7: System efficiency with respect to the number of tags, given $K=30, L=32, L_{\mathrm{c}}=31, \mathrm{SNR}=7 \mathrm{~dB}$.

We now validate our analysis in the subsection 3.2 by showing the theoretical and simulation results of the considered system efficiency $\eta$ with QDD, for a given number tags in Figure 6. The frame size $L$ and the code length $L_{\mathrm{c}}$ are supposedly to be 32 and 31, respectively. We can see that the results are matched to each other, that confirms the correctness of the analysis.

Under the effect of the detection error and code collision, the system efficiency $\eta$ with both QDD and $\mathrm{DD}$ is evaluated with respect to the number of tags and the number of codes in Figures 7 and 8, respectively. Here, it is noted that if one among 96 bits of a tag's ID is wrongly detected, it is not decoded successfully. We can see in both figures that the system efficiency with QDD is larger than that with DD (especially when the number of codes increases as in Figure 8). The reason, which has been mentioned above, is the noise

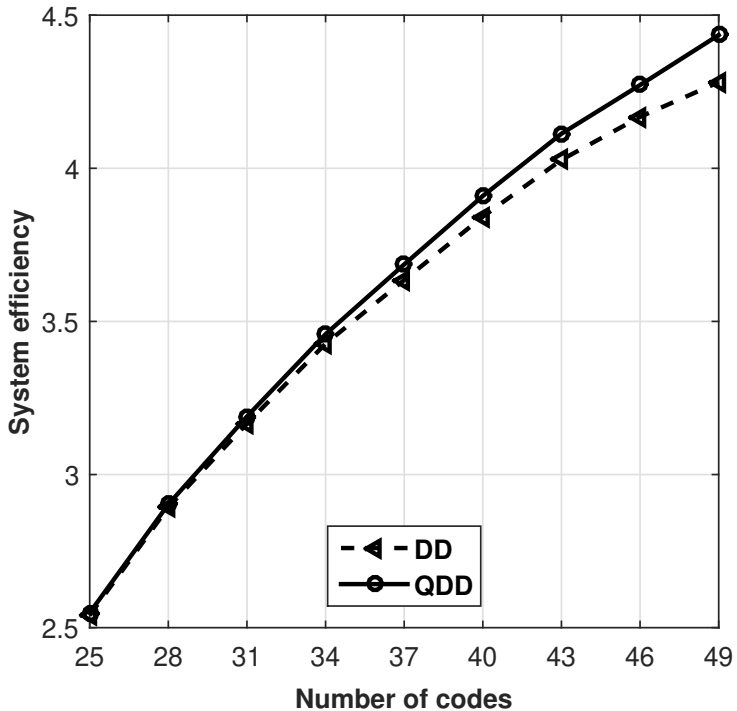

Figure 8: System efficiency with respect to the number of codes, given $K=30, L=32, L_{\mathrm{c}}=31, \mathrm{SNR}=7 \mathrm{~dB}$.

enhancement in DD caused by the code correlation matrix $\mathbf{R}^{-1}$, and the noise mitigation in QDD thanks to the stage truncation of the inverse of the correlation matrix $\mathbf{M}_{r}$. The results confirm the advantages of QDDbased detector in the structure of RFID readers in comparison with the DD-based one.

The system efficiency with respect to different values of the frame size is re-plotted in Figure 9, for given $N=1000, K=30, L_{\mathrm{c}}=31$, and different values of SNR $(\mathrm{SNR}=5 \mathrm{~dB}$ in Figure 9(a) and $7 \mathrm{~dB}$ in Figure 9(b)). It is interesting to see that for a given value of SNR, we can choose an optimal frame size that maximize the system efficiency. In our examples, the optimal frame sizes are 35 and 30 time slots. This fact might suggest a suitable selection of system parameters for the identification process in practical systems, which we believe very useful for system designers.

\subsection{False Alarm and False Detection}

We now evaluate the performance of the conventional missing-tag protocol proposed in [21] with QDD. Two performance metrics i.e. false alarm rate and false detection rates, denoted by $R_{\mathrm{fa}}$ and $R_{\mathrm{fd}}$, respectively, are presented. In particular, false alarm occurs when an available tag in system is notified missing, and thus, the rate is defined as follows

$$
R_{\mathrm{fa}}=\frac{N_{\mathrm{fa}}}{N},
$$

where $N_{\mathrm{fa}}$ is the number of available tags detected as missing ones. On the other hand, false detection occurs when an actual missing tag is confirmed to be present in systems and thus, the rate $R_{\mathrm{fd}}$ is defined as

$$
R_{\mathrm{fd}}=\frac{N_{\mathrm{fd}}}{N}
$$

where $N_{\mathrm{fd}}$ is the number of actual missing tags detected as available ones. 


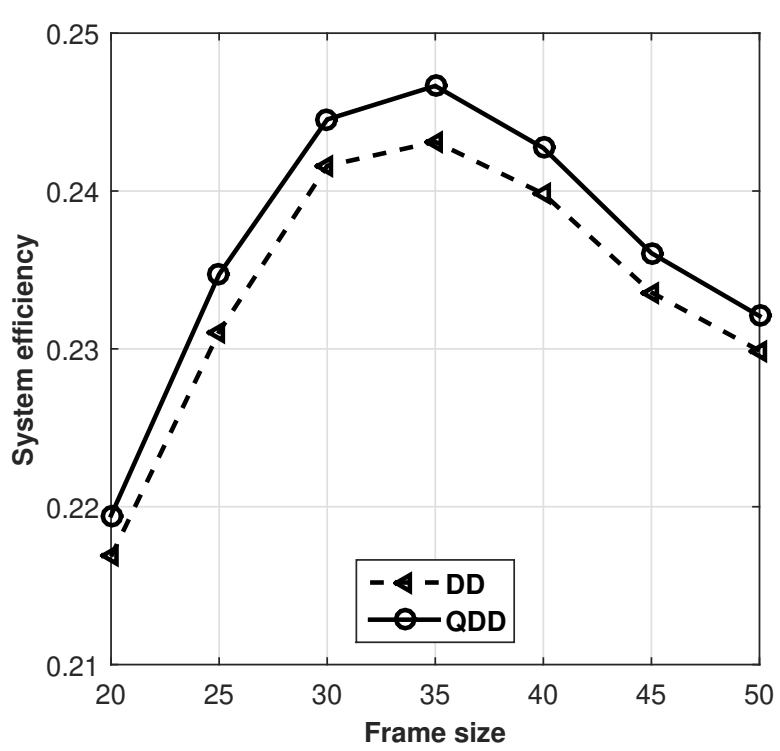

(a) $\mathrm{SNR}=5 \mathrm{~dB}$

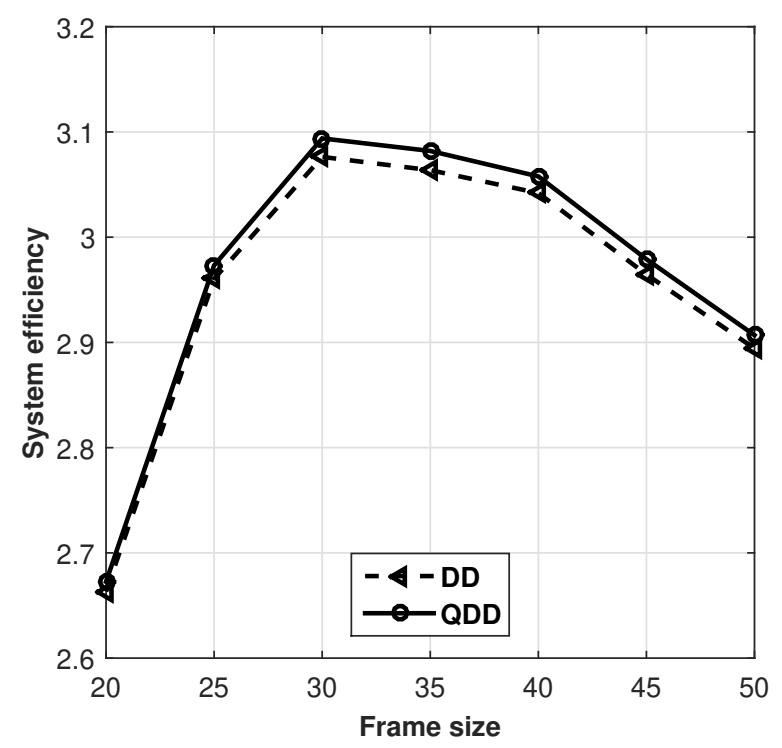

(b) $\mathrm{SNR}=7 \mathrm{~dB}$

Figure 9: System efficiency with respect to frame size, given $N=1000, K=30, L_{\mathrm{c}}=31$.

We specifically plot in Figure 10(a) the false alarm rate and Figure 10(b) the false detection rate with respect to different values of SNR. In the figures, $N$, $L, K, m$, and threshold for detection are set to be 1000, $512,15,4$, and 0.3 , respectively. Here, it is noted that the threshold is used to detect the transmitted binary bit (i.e., 0/1) from tags. We can see that, thanks to the efficiency of QDD in coping with noisy channels, the rates with QDD are lower than those with DD, and they will be most the same when the SNR keeps increasing. This is because when SNR increases, the detection error decreases, and thus, the performance of the protocol is more reliable regardless of the detector.

Finally, we plot in Figure 11 the rates versus the threshold where $N=1000, L=512, K=15, m=4$, and $\mathrm{SNR}=0 \mathrm{~dB}$. Again, we observe that the reliability

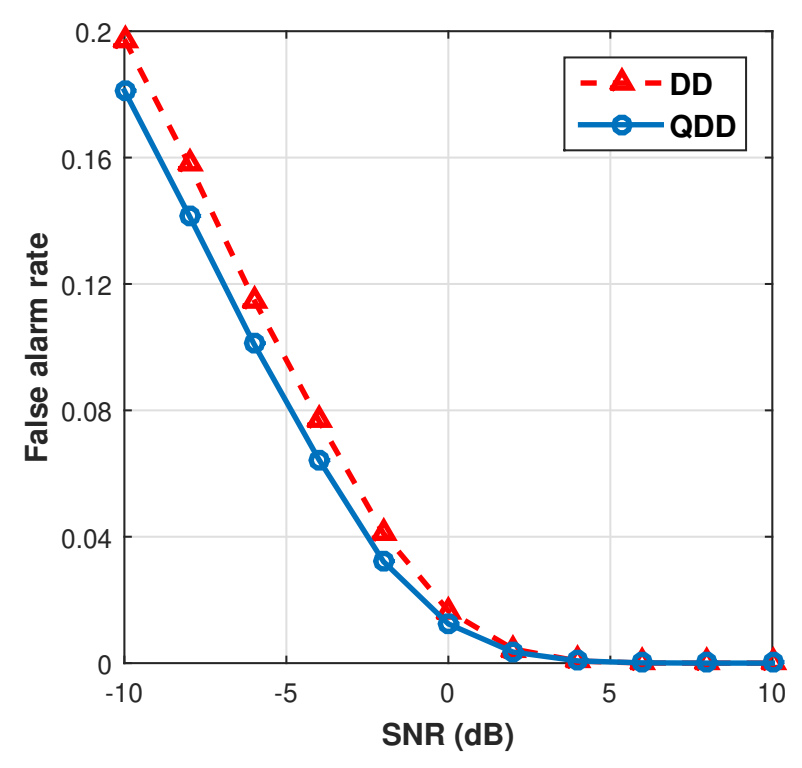

(a) False alarm rate

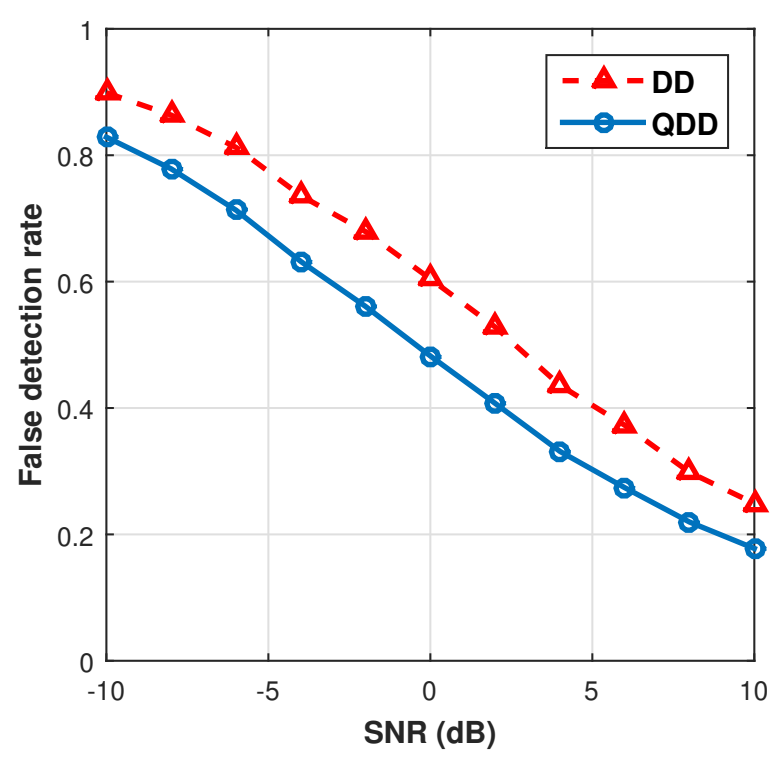

(b) False detection rate

Figure 10: False alarm and false detection rate with respect to the SNR in the conventional missing-tag detection protocols with DD and QDD, given $N=1000$, $K=15, L=512, m=4$, Threshold $=0.3$.

of missing-tag detection protocol with QDD is mostly better than that with DD. Based on this simulation results, system designers might have an optimal protocol parameters setting depending on practical transmission environments.

\section{Conclusions}

In this paper, we have investigated the performance of a hybrid ALOHA/CDMA RFID system with both QDD and DD. The structure and the performance of QDD and DD were re-studied in the context of RFID. The system efficiency was then re-analyzed in practical 


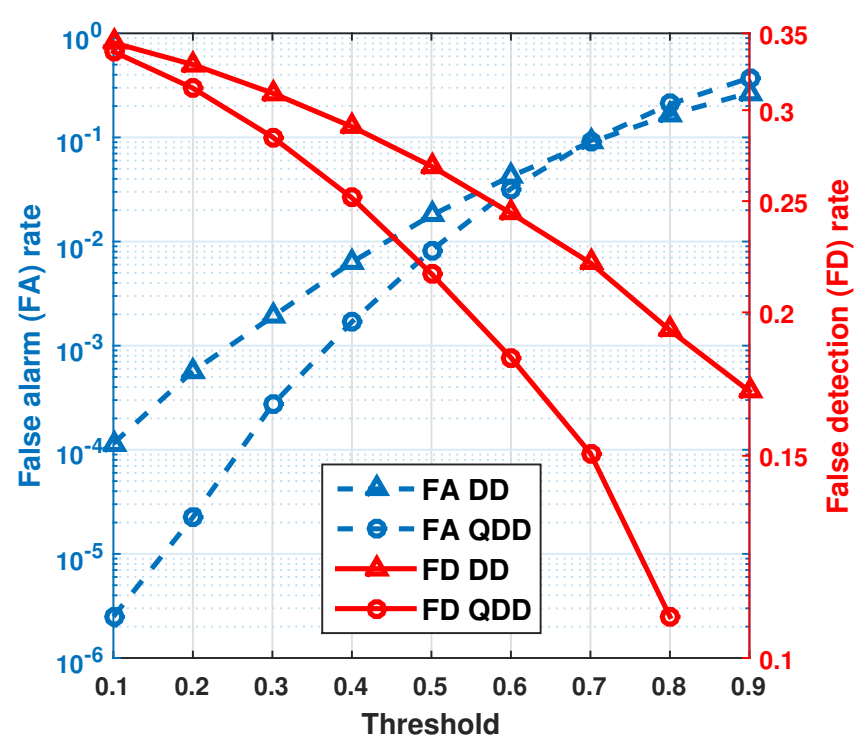

Figure 11: False alarm and false detection rates with respect to the threshold in the conventional missing-tag detection protocols with DD and QDD, given $N=1000$, $K=15, L=512, m=4, \mathrm{SNR}=0 \mathrm{~dB}$.

environments with the presence of both code collision and detection error. Computer simulations were performed, which showed that the analytical efficiency matched with the simulation one. It was also observed that identification and missing-tag detection protocols with QDD outperformed those with DD in practical noisy channels, which we believed useful for system designers.

\section{ACKNOWLEDGMENT}

This work is supported by Hanoi University of Science and Technology, Vietnam, through a project number T2017-PC-115.

\section{REFERENCES}

[1] S. A. Ahson and M. Ilyas, RFID Handbook: Applications, Technology, Security, and Privacy. CRC press, 2008.

[2] M. Bolic, D. Simplot-Ryl, and I. Stojmenovic, RFID systems: Research trends and Challenges. John Wiley \& Sons, 2010.

[3] L. Arjona, H. Landaluce, A. Perallos, and E. Onieva, "Timing-aware RFID anti-collision protocol to increase the tag identification rate," IEEE Access, 2018.

[4] L. Zhang, W. Xiang, X. Tang, Q. Li, and Q. Yan, "A timeand Energy-Aware collision tree protocol for efficient large-scale RFID tag identification," IEEE Transactions on Industrial Informatics, vol. 14, no. 6, pp. 2406-2417, 2018.

[5] M. Shahzad and A. X. Liu, "Fast and reliable detection and identification of missing RFID tags in the wild," IEEE/ACM Transactions on Networking, vol. 24, no. 6, pp. 3770-3784, 2016.

[6] M. A. Razzaque, M. Milojevic-Jevric, A. Palade, and S. Clarke, "Middleware for Internet of Things: A survey." IEEE Internet of Things Journal, vol. 3, no. 1, pp. 70-95, 2016.

[7] J. Lin, W. Yu, N. Zhang, X. Yang, H. Zhang, and W. Zhao, "A survey on internet of things: Architecture, enabling technologies, security and privacy, and applications,"
IEEE Internet of Things Journal, vol. 4, no. 5, pp. 11251142, 2017.

[8] C. T. Nguyen, T. T. Hoang, and V. X. Phan, "A simple method for anonymous tag cardinality estimation in RFID systems with false detection," in Proceedings of the 4th NAFOSTED Conference on Information and Computer Science. IEEE, 2017, pp. 101-104.

[9] D. K. Klair, K.-W. Chin, and R. Raad, "A survey and tutorial of RFID anti-collision protocols," IEEE Communications Surveys $\mathcal{E}$ Tutorials, vol. 12, no. 3, pp. 400-421, 2010.

[10] H. Landaluce, A. Perallos, E. Onieva, L. Arjona, and L. Bengtsson, "An energy and identification time decreasing procedure for memoryless RFID tag anticollision protocols," IEEE Transactions on Wireless Communications, vol. 15, no. 6, pp. 4234-4247, 2016.

[11] A. D. Smith, A. A. Smith, and D. L. Baker, "Inventory management shrinkage and employee anti-theft approaches," International Journal of Electronic Finance, vol. 5, no. 3, pp. 209-234, 2011.

[12] M. S. Humphreys, "Shoplifting-in 100 words," British Journal of Psychiatry, vol. 202, no. 3, p. 128, 2013.

[13] W. Su, N. Alchazidis, and T. T. Ha, "Multiple RFID tags access algorithm," IEEE Transactions on Mobile Computing, vol. 9, no. 2, pp. 174-187, 2010.

[14] C. Mutti and C. Floerkemeier, "CDMA-based RFID systems in dense scenarios: Concepts and Challenges," in Proceedings of the IEEE International Conference on RFID. IEEE, 2008, pp. 215-222.

[15] A. F. Mindikoglu and A.-J. van der Veen, "Separation of overlapping RFID signals by antenna arrays," in Proceedings of the IEEE International Conference on Acoustics, Speech and Signal Processing (ICASSP), 2008, pp. 27372740.

[16] J. Yu, K. H. Liu, X. Huang, and G. Yan, "An anti-collision algorithm based on smart antenna in RFID system," in Proceedings of the International Conference on Microwave and Millimeter Wave Technology (ICMMT), vol. 3. IEEE, 2008, pp. 1149-1152.

[17] K. Finkenzeller, RFID handbook: Fundamentals and Applications in Contactless Smart Cards and Identification, 2003.

[18] C. T. Nguyen, K. Hayashi, M. Kaneko, and H. Sakai, "Maximum likelihood approach for RFID tag cardinality estimation under capture effect and detection errors," IEICE Transactions on Communications, vol. 96, no. 5, pp. 1122-1129, 2013.

[19] T. F. L. Porta, G. Maselli, and C. Petrioli, "Anticollision protocols for single-reader RFID systems: Temporal analysis and optimization," IEEE Transactions on Mobile Computing, vol. 10, no. 2, pp. 267-279, Feb 2011.

[20] C. T. Nguyen, T. C. Thang, and A. T. Pham, "Performance analysis of Hybrid ALOHA/CDMA anti-collision scheme for RFID systems over fading channels," in Proceedings of the Seventh International Conference on Ubiquitous and Future Networks (ICUFN). IEEE, 2015, pp. 657-661.

[21] C. T. Nguyen, V. X. Phan, N. X. Quyen, T. M. Hoang, and A. T. Pham, "Performance evaluation of missing-tag algorithms in CDMA-based RFID systems," in Proceedings of the IEEE Sixth International Conference on Communications and Electronics (ICCE). IEEE, 2016, pp. 131-135.

[22] R. Corvaja and S. Pupolin, "Multi-user performance of CDMA in the presence of phase noise," in Proceedings of the Global Telecommunications Conference (GLOBECOM), vol. 6. IEEE, 1998, pp. 3314-3319.

[23] H.-H. Chen, “Quasi-decorrelating detector: A non-matrix inversion based decorrelating detector with near-far resistance and complexity trade-off," European Transactions on Telecommunications, vol. 16, no. 4, pp. 273-289, 2005.

[24] Z. Zhang, Z. Lu, Q. Chen, and X. Yan, "Design and optimization of a CDMA-based multi-reader passive UHF RFID system for dense scenarios," IEICE Transactions on Communications, vol. 95, no. 1, pp. 206-216, 2012. 
[25] E. EPCglobal, “Radio-frequency identity protocols Class1 Generation-2 UHF RFID protocol for communications at $860 \mathrm{Mhz}-960 \mathrm{Mhz}$ version 1.0. 9," K. Chiew et al./On False Authenticationsfor C1G2 Passive RFID Tags, vol. 65, 2004.

[26] R. Lupas and S. Verdú, "Linear Multiuser Detectors for synchronous Code Division Multiple Access channels," IEEE Transactions on Information Theory, vol. 35, no. 1, pp. 123-136, 1989.

[27] L.-S. Shieh, X. Zou, and J. S. Tsai, “Model conversion of continuous-time uncertain systems via the interval geometric-series method," IEEE Transactions on Circuits and Systems I: Fundamental Theory and Applications, vol. 43, no. 10, pp. 851-854, 1996.

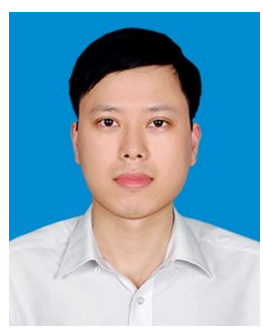

Hoang Trung Tuyen received his B.S. and M.S. degrees in Telecommunications from Hanoi University of Science and Technology (HUST), Vietnam, in 2012 and 2015, respectively. He is currently pursuing his Ph.D. degree at School of Electronics and Telecommunication, HUST. His research interests are in areas of protocol design for RFID-based systems.

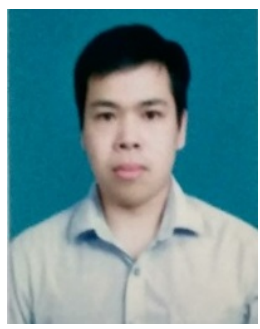

Dao Van Hieu is currently an undergraduate student at School of Electronics and Telecommunications, Hanoi University of Science and Technology, Vietnam. His research interests are in areas of protocol design for RFID-based systems.

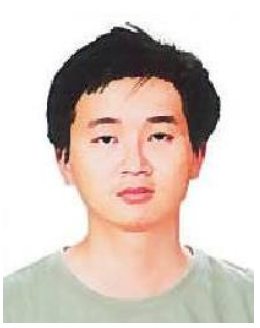

Phan Xuan Vu received the B.S. degree in computer science and the M.S. degree in Intelligent and Communication Systems from the University of Cergy-Pontoise, France, in 2008 and 2010, respectively, and the Ph.D. degree in Signal Processing from the University of Rennes 1 and University of Grenoble, France, in 2014. He is currently working as a lecturer-researcher in the School of Electronics and Telecommunications, Hanoi University of Science and Technology, Vietnam. His research interests include microwave engineering, antenna and propagation, radar signal processing, and large-scale RFID.

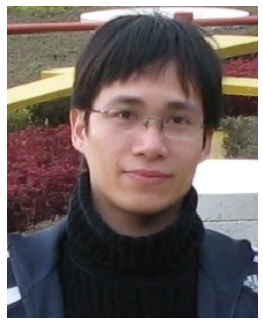

Nguyen Thanh Chuyen received his B.E. degree in Electronics and Telecommunications, Hanoi University of Science and Technology (HUST), Vietnam in 2006, M.E. degree in Communications Engineering from National TsingHua University, Taiwan in 2008, and Ph.D. degree in Informatics from Kyoto University, Japan in 2013. From September to November 2014, he was a visiting researcher in The University of Aizu, Japan. He received the Fellow award from the Hitachi Global Foundation in August 2016. He is currently an Assistant Professor at School of Electronics and Telecommunications, HUST, Vietnam. His current research interests are in areas of communications theory and applications with a particular emphasis on RFID-based systems. 